\title{
Quantum Correction to Conductivity Close to Ferromagnetic Quantum Critical Point in Two Dimensions
}

\author{
I. Paul ${ }^{1}$, C. Pépin ${ }^{1}$, B. N. Narozhny ${ }^{2}$, and D. L. Maslov ${ }^{3}$ \\ ${ }^{1}$ SPhT, CEA-Saclay, L'Orme des Merisiers, 91191 Gif-sur-Yvette, France \\ ${ }^{2}$ The Abdus Salam ICTP, Strada Costiera 11, Trieste, 34100, Italy \\ ${ }^{3}$ Dept. of Physics, University of Florida, P. O. Box 118440, Gainesville, FL 32611-8440, USA
}

(Dated: November 8, 2018)

\begin{abstract}
We study the temperature dependence of the conductivity due to quantum interference processes for a two-dimensional disordered itinerant electron system close to a ferromagnetic quantum critical point. Near the quantum critical point, the cross-over between diffusive and ballistic regimes of quantum interference effects occurs at a temperature $T^{*}=1 / \tau \gamma\left(E_{F} \tau\right)^{2}$, where $\gamma$ is the parameter associated with the Landau damping of the spin fluctuations, $\tau$ is the impurity scattering time, and $E_{F}$ is the Fermi energy. For a generic choice of parameters, $T^{*}$ is smaller than the nominal crossover scale $1 / \tau$. In the ballistic quantum critical regime, the conductivity behaves as $T^{1 / 3}$.

PACS numbers: $75.45 .+\mathrm{j}, 72.15 .-\mathrm{v}, 72.15 . \mathrm{Rn}$
\end{abstract}

The interplay between disorder, electron correlations, and low dimensionality is one of the most fascinating topics in the modern condensed matter. To date, most of the studies were limited to the case of "good metals" which, at high enough temperatures, behave as Fermi Liquids (FL) [1, 2, 3]. However, this interplay is expected to become crucial in the vicinity of a quantum critical point (QCP) where electron correlations are particularly strong [4, 5]. Experiments on systems close to quantum phase transitions show striking deviations from the FL theory. In particular, anomalous exponents in the temperature dependence of the conductivity have been observed [6, 7], which suggest the presence of strong quantum fluctuations. Of special interest is the case of charge transport in the vicinity of a ferromagnetic QCP. Since ferromagnetic spin fluctuations do not break any lattice symmetry, the contribution of inelastic scattering to resistivity is zero in a clean system, unless Umklapp processes are allowed to relax momentum. In a dirty system, the "interaction" correction to the residual conductivity is expected to be particularly important due to a long-range interaction in the vicinity of the QCP. This correction is due to quantum interference between semi-classical electron paths scattered by the impurities and the self-consistent potential of Friedel oscillations [2]. The goal of this paper is to examine the conductivity of a two-dimensional (2D) disordered metal close to a ferromagnetic QCP and at low enough temperatures, when the lattice-mediated scattering at spin fluctuations is frozen out and the temperature dependence of the conductivity is mainly due to quantum interference effects.

The experiments indicate that most of the threedimensional compounds, such as $\mathrm{UGe}_{2}$ [6] and $\mathrm{ZrZn}_{2}$ [7], undergo a first-order zero-temperature ferromagnetic transition. More recently, the transition observed in $\mathrm{Zr}_{1-x} \mathrm{Nb}_{x} \mathrm{Zn}_{2}$ is found to be second order down to the lowest measured transition temperature [8]. In two di- mensions, the best candidate for a ferromagnetic type of quantum critical behavior is the metamagnetic transition in $\mathrm{Sr}_{3} \mathrm{Ru}_{2} \mathrm{O}_{7}$ [9, 10]. This strongly anisotropic compound can be tuned to a quantum critical end point which is believed to be suitable for a description within the spin fluctuation scenario 11]. Transport properties of disordered metallic systems are well understood in the case when the electron-electron interaction is weak enough (so that the system is away from any QCP and the symmetries of the FL state are not broken) [1, 12]. At low enough temperature $(T)$, the $T$-dependence of the conductivity (as well as other transport coefficients) is mostly due to quantum interference [1]. The effect is more dramatic in lower dimensions, where the temperature dependent corrections to the residual conductivity exhibit singular behavior. In particular, in 2D the corrections are logarithmic in the diffusive regime, when $T \tau \ll 1[\mathbb{1}$, and linear in the ballistic regime $T \tau \gg 1[3]$, where $\tau$ is the elastic scattering lifetime of the electrons. Quantum correction to conductivity has also been studied in the context of fermion gauge field models [13, 14].

Near a QCP the interaction between electrons is strong, making it difficult to formulate a controlled theory. Therefore it is not surprising that there has been very few studies of transport properties near quantum criticality 15, 16, 17]. For a metamagnetic QCP in 2D it has been shown [17] that the conductivity in the diffusive regime behaves as $\ln ^{2} T$, in contrast to the usual logarithmic temperature dependence in a good metal [1] .

In this Letter we study the conductivity $(\sigma)$ of a disordered 2D metal near a ferromagnetic QCP, assuming the system to be in a continuum where lattice effects are absent. In the conventional approach to QCP [18], the conduction electrons are integrated out, and a generalized Landau-Ginzburg action in terms of the order parameter fields is studied. Recently, the validity of integrating out low-energy electrons has been questioned 19, 20], and it has been argued that such an approach gener- 
ates singularities to all orders in the collective spin interactions. Here, we start with the phenomenological spin-fermion model of Ref. 21], which describes the lowenergy properties of electrons close to a ferromagnetic instability, and add scattering of electrons due to static impurities. For completeness, we also take into account the coupling to the long-range Coulomb interaction in the singlet (charge) channel, so that the total correction to the conductivity is the sum of singlet and triplet contributions: $\delta \sigma(T)=\delta \sigma_{S}(T)+\delta \sigma_{T}(T)$. Both in the diffusive and ballistic regimes, $\delta \sigma_{S}(T)$ has an insulating-like behavior common to all metals [3], which competes with the metallic-like behavior of $\delta \sigma_{T}(T)$. Since the interaction in the triplet channel is enhanced near the QCP, $\delta \sigma_{T}(T)$ is expected to be larger than $\delta \sigma_{S}(T)$-which is what we find in almost all regimes of interest. On the other hand, we disregard the weak-localization correction, which is not relevant for metamagnetic transitions and can readily be accounted for otherwise. The correction in the triplet channel $\delta \sigma_{T}(T)$ is calculated within the spin-fermion model of Ref. 21]. The interaction in this model can be treated perturbatively if $\gamma \gg \alpha$, where $\gamma$ is the dimensionless parameter associated with Landau damping of the spin fluctuations, and $\alpha$ is the dimensionless coupling between the electrons and the spin fluctuations. While this relation holds, we are able to study the various cross-over regimes in the entire $T-\delta$ plane (where $\delta$ is the distance from the QCP) down to very low temperature. What is new in our study is that (1) we identify the regime of parameters in which controlled calculations are possible in the entire $T-\delta$ plane, (2) we find a new power law dependence $\left(\delta \sigma \propto-T^{1 / 3}\right)$ of the conductivity in the ballistic quantum critical regime, and (3) near the QCP we find the temperature scale of ballistic-diffusive cross-over to be much smaller than the nominal scale $1 / \tau$ :

$$
T^{*}=1 /\left(\tau\left(E_{F} \tau\right)^{2} \gamma\right) \ll 1 / \tau .
$$

The model. - We describe the system by the action

$$
\begin{aligned}
S & =T \sum_{\omega_{n}} \int d^{2} r \psi_{\alpha}^{\dagger}\left(\mathbf{r}, \omega_{n}\right)\left(i \omega_{n}+\nabla^{2} / 2 m+\mu\right) \psi_{\alpha}\left(\mathbf{r}, \omega_{n}\right) \\
& +\left(E_{0} T\right) \sum_{\Omega_{n}, \mathbf{q}} U^{-1}\left(\mathbf{q}, \Omega_{n}\right) \mathbf{S}\left(\mathbf{q}, \Omega_{n}\right) \cdot \mathbf{S}\left(-\mathbf{q},-\Omega_{n}\right) \\
& +\left(\alpha E_{0} / \nu\right)^{1 / 2} \int d^{2} r \int_{0}^{\beta} d \tau \psi_{\alpha}^{\dagger}(\mathbf{r}, \tau) \psi_{\beta}(\mathbf{r}, \tau)\left[\mathbf{S}(\mathbf{r}, \tau) \cdot \sigma_{\alpha \beta}\right] \\
& +\int_{0}^{\beta} d \tau \int d^{2} r \psi_{\alpha}^{\dagger}(\mathbf{r}, \tau) V(\mathbf{r}) \psi_{\alpha}(\mathbf{r}, \tau)
\end{aligned}
$$

where summation over repeated indices is implied. Here $\left(\psi_{\alpha}^{\dagger}, \psi_{\alpha}\right)$ are Grassman fields for (low-energy) electrons with spin $\alpha, \mathbf{S}\left(\mathbf{q}, \Omega_{n}\right)$ is a bosonic field for the collective spin fluctuation modes, $E_{0}$ has dimension of energy, $\nu=m / \pi$ is the density of states for non-interacting electrons with spin in $2 \mathrm{D}$, and $\mu$ is the chemical potential.
Fields $\mathbf{S}\left(\mathbf{q}, \Omega_{n}\right)$ are obtained by integrating out electrons above a certain energy cut-off (for example below which the electron spectrum can be linearized). The disorder potential $V(\mathbf{r})$ is assumed to obey Gaussian distribution with $\left\langle V\left(\mathbf{r}_{1}\right) V\left(\mathbf{r}_{2}\right)\right\rangle=\delta\left(\mathbf{r}_{1}-\mathbf{r}_{2}\right) /(2 \pi \nu \tau)$. In our theory the dimensionless coupling constant $\alpha \lesssim 1$.

In the ballistic regime the propagator for the spin fluctuations is

$$
U\left(\mathbf{q}, \Omega_{n}\right)=\left[\delta+\left(q / p_{F}\right)^{2}+\gamma\left|\Omega_{n}\right| / v_{F} q\right]^{-1},
$$

where $\delta$ is related to the magnetic correlation length $\xi$ by $\delta=\left(p_{F} \xi\right)^{-2}$. Although the dimensionless parameter $\gamma$ is not unrelated to the coupling $\alpha$ (for example, $\gamma$ should vanish when $\alpha$ is zero), the precise relation between the two depends on microscopic details. In the random phase approximation, $\gamma=\alpha$ 21]. In our theory we take $\gamma$ as an independent phenomenological parameter. The form of the Landau-damping term in Eq. (3) is valid for $v_{F} q \gg \Omega$, where it is a universal low-energy feature of itinerant electrons [22]. In the opposite limit of $\Omega \gg v_{F} q$, the Landau-damping term depends on microscopic details, and the spin-fermion model loses universality. We find that in the ballistic regime either $v_{F} q \gg \Omega$ (thus justifying the universal form of the Landau damping), or the contribution of the dynamic term in Eq. (3) is negligible to leading order. In this sense our results are universal. In the diffusive limit, the phenomenological form of the spin fluctuation propagator is given by replacing the dynamic term in Eq. (3) by $\gamma\left|\Omega_{n}\right| / D q^{2}$, where $D=v_{F}^{2} \tau / 2$ is the diffusion constant.

Near the QCP, there are two important temperature scales. (i) The temperature scale $T^{*}[13$ ] of the crossover between ballistic and diffusive motion of the electrons. The cross-over occurs when the distance travelled by an electron during interaction, which by uncertainty relation is $1 / q$ for momentum transfer $q$, is comparable to the distance $v_{F} \tau$ travelled by electrons between successive impurity scatterings. Very close to the QCP $\left(\delta \ll\left(E_{F} \tau\right)^{-2}\right)$, the momentum transfer $q_{B 1} \sim$ $p_{F}\left(\gamma \Omega / E_{F}\right)^{1 / 3}$ is determined by the pole of the propagator in Eq. (3). Since $\Omega \sim T$, we get the cross-over scale $T^{*}$ in Eq. (11). In the FL-regime far away from the QCP $(\delta \gg \gamma), q$ is of order of the typical momentum of fermionic excitations $q_{F} \sim \Omega / v_{F}$, and the ballisticdiffusive cross-over scale is $1 / \tau$. In the FL-regime close to the QCP $\left(\left(E_{F} \tau\right)^{-2} \ll \delta \ll \gamma\right), q \sim q_{B 2} \sim(\gamma \Omega) /\left(v_{F} \delta\right)$ is still controlled by the pole in Eq. (3), and the ballisticdiffusive cross-over scale is $\delta /(\gamma \tau)$. (ii) $T_{1}=\gamma^{1 / 2} E_{F}$ is the scale above which $q_{F} \gg q_{B 1}$, and the effect of the QCP on the conductivity is small. We identify two possible situations depending on the strength of disorder relative to the Landau damping parameter. (a) For $\gamma^{1 / 2}>1 /\left(E_{F} \tau\right)$, the low-temperature cut-off of the regime where $\delta \sigma \propto-T^{1 / 3}$ is $T^{*}$ and the high-T cut-off is $T_{1}$ (see Fig. 1). For $T<T^{*}$, we recover the result of 
Ref. 17] with $\delta \sigma_{T} \propto \ln ^{2}(T)$ (however, the (metallic) sign of our result is opposite to that in Ref. 17] ). Above $T_{1}$ the correction in the triplet channel $\delta \sigma_{T} \propto 1 / T$ is smaller than the singlet-channel one and $\delta \sigma \approx \delta \sigma_{S}=e^{2} T \tau / \pi[3]$. (b) For $1 /\left(E_{F} \tau\right)>\gamma^{1 / 2}$, a situation which is experimentally highly improbable, the $T^{1 / 3}$ regime is lost.

The $T^{1 / 3}$ scaling of $\delta \sigma_{T}$ in the ballistic quantum critical regime in $2 \mathrm{D}$ can be simply understood from the following argument. The correction to the scattering rate due to electron-electron interaction can be estimated as $\Delta[1 / \tau] \sim(1 / \tau) \operatorname{Im} \Sigma \Delta t$, where $\operatorname{Im} \Sigma$ is determined by the interaction between the electrons mediated by the spin fluctuations, and $\Delta t$ is the interaction time. By uncertainty principle, $\Delta t \sim 1 / v_{F} q$. In the FL-regime, $\operatorname{Im} \Sigma \propto T^{2}$ and $q \propto \Omega \sim T$, hence $\Delta[1 / \tau] \propto T$. In $2 \mathrm{D}$ near the $\mathrm{QCP}$, and an interaction with a dynamical exponent $z$ (in our case $z=3$ in the ballistic regime, see Eq. (3) $), \operatorname{Im} \Sigma \propto T^{(1-1 / z)}\left[23\right.$ and $q \propto \Omega^{1 / z} \propto T^{1 / z}$, hence $\Delta[1 / \tau] \propto T^{(1-2 / z)}$.

We summarize the technical details [3] of the intermediate steps. First, using Kubo formalism we expand the current-current correlator to the lowest order in $\alpha$. In the ballistic regime near the $\mathrm{QCP}$, the vertex correction to the spin-fermion coupling gives contribution which is smaller by a factor $\left(\alpha / \gamma^{1 / 2}\right)\left(T / T_{1}\right)^{1 / 3} \ln \left(T_{1} / T\right)$ for $T \ll T_{1}$, and by a factor $\alpha / \gamma^{1 / 2}$ for $T \gg T_{1}$. In the diffusive regime the next order in coupling $\alpha$ is smaller by $\alpha \ln ^{2}(T) /\left(E_{F} \tau\right)$. As a result, the expansion in the coupling constant $\alpha$ is controlled. The second step is to perform the analytic continuation. In the third step we average over disorder. The correction to the conductivity in the triplet channel can be written as 3 ]

$$
\begin{aligned}
\delta \sigma_{T} & =-\left(3 \pi e^{2} v_{F}^{2} \tau \alpha\right) \int_{-\infty}^{\infty} \frac{d \Omega}{4 \pi^{2}}\left[\frac{\partial}{\partial \Omega}\left(\Omega \operatorname{coth} \frac{\Omega}{2 T}\right)\right] \\
& \times \operatorname{Im} \int \frac{d^{2} q}{(2 \pi)^{2}} U^{A}(\mathbf{q}, \Omega) B(\mathbf{q}, \Omega),
\end{aligned}
$$

where $B(\mathbf{q}, \Omega)$ is the fermionic part of the current-current correlator (see Eq. (3.26) in Ref. [3]). In the ballistic regime $v_{F} q \gg 1 / \tau$, and the limiting form of $B$ is given by the term leading in $\tau$. This is equivalent to an expansion in $\left(T / T^{*}\right)^{1 / 3}$ near the QCP, and in $1 /(T \tau)$ for $\delta \gg \gamma$. In this limit $B \approx B_{b}$, where $B_{b}$ is given by

$$
B_{b}(\mathbf{q}, \Omega)=\left(2 /\left(v_{F} q\right)^{2}\right)(1-(i \Omega) / S)^{2}+\left(2 / S^{2}\right)(1-(i \Omega) / S),
$$

where $S=\left(\left(v_{F} q\right)^{2}-\Omega^{2}+i \eta \operatorname{Sgn}(\Omega)\right)^{1 / 2}$. In the diffusive regime $v_{F} q \ll 1 / \tau$, and the typical momentum is given by the diffusion pole. In this limit $B \approx B_{d}$, where

$$
B_{d}(\mathbf{q}, \Omega)=\left(\tau\left(v_{F} q\right)^{2}\right) /\left(i \Omega+D q^{2}\right)^{3} .
$$

Results. - The ballistic limit is defined by $T \gg T^{*}$ for $\delta \ll\left(E_{F} \tau\right)^{-2}$, by $T \gg \delta / \gamma \tau$ for $\left(E_{F} \tau\right)^{-2} \ll \delta \ll \gamma$, and by $T \gg 1 / \tau$ for $\delta \gg \gamma$. In this limit, there are three cross-over regimes (regions I-III in Fig. (1).

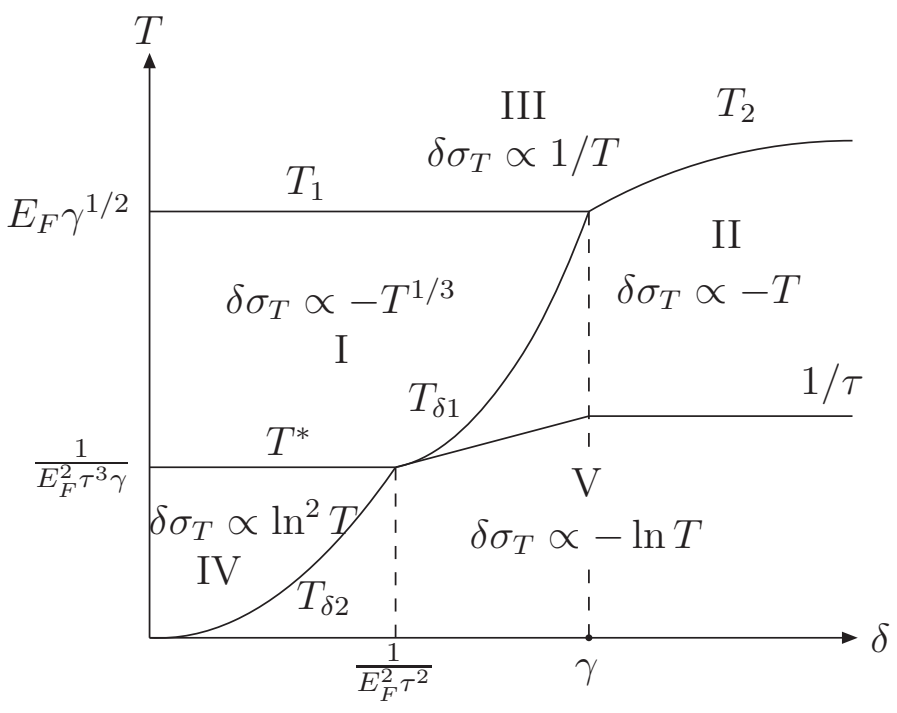

FIG. 1: Different cross-over regimes for the temperature dependence of the triplet channel contribution to conductivity. $T_{\delta 1}=\left(\delta^{3 / 2} / \gamma\right) E_{F}, T_{\delta 2}=\left(\delta^{2} \tau / \gamma\right) E_{F}^{2}, T_{2}=E_{F} \delta^{1 / 2}$. Notice that $\gamma^{1 / 2} \gg 1 /\left(E_{F} \tau\right)$.

(1) Regime I. The limiting form of $U$ is obtained by setting $\delta=0$ in Eq. (3), which gives the bosonic momentum scale $q_{B 1} \sim p_{F}\left(\gamma \Omega / E_{F}\right)^{1 / 3}$. This is the momentum transferred by the spin fluctuations to the electrons during elastic scattering. In this regime, $q_{B 1} \gg q_{F} \sim \Omega / v_{F}$. Since, $v_{F} q_{B 1} \gg \Omega$, the form of $B_{b}$ simplifies to $B_{b} \approx$ $4 /\left(v_{F} q\right)^{2}$. The leading temperature dependence of the conductivity is given by the triplet channel contribution

$$
\delta \sigma_{T}(T)=-\frac{e^{2} \tau \alpha}{\pi \gamma^{2 / 3}} \mathcal{C}\left(p_{F} v_{F}\right)^{2 / 3} T^{1 / 3}
$$

where $\mathcal{C}=\int_{0}^{\infty} d t \frac{\partial}{\partial t}\left(\frac{2 t}{1-e^{t}}\right) \frac{1}{t^{2 / 3}} \approx 3.44$. Eq. (4) is the main result of this Letter. The high temperature cutoff of this regime is $T_{1}$, above which fermionic momentum $q_{F} \gg q_{B 1}$. At finite $\delta$ the regime ends when $\delta \sim\left(q_{B 1} / p_{F}\right)^{2}$. This gives the cross-over scale $T_{\delta 1}=$ $E_{F} \delta^{3 / 2} / \gamma$. For temperature below $T_{\delta 1}$ the effect of finite $\delta$ is important.

(2) Regime II. Two situations can be identified in this regime. For $\delta \ll \gamma$, the approximate form of $U$ is given by dropping the $\left(q / p_{F}\right)^{2}$-term in Eq. (3). The dominant momentum scale is $q_{B 2} \sim(\gamma \Omega) /\left(v_{F} \delta\right) \gg q_{F}$, and $B_{b} \approx$ $4 /\left(v_{F} q\right)^{2}$. For $\delta \gg \gamma$, the typical momentum scale is given by $q_{F} \sim \Omega / v_{F}$. In this limit the Landau damping term is order $\gamma \ll \delta$, and so $U \approx 1 / \delta$. For both cases the triplet channel contribution is

$$
\delta \sigma_{T}(T)=-\left(3 e^{2} \tau \alpha / \pi \delta\right) T .
$$

For $\delta \gg \gamma$, this regime is cut-off at $T_{2}=\delta^{1 / 2} E_{F}$, above which $\left(q / p_{F}\right)^{2}$ term in $U$ dominates since $\left(q / p_{F}\right)^{2} \sim$ $\left(\Omega / E_{F}\right)^{2} \gg \delta$. 
(3) Regime III. This is the high temperature regime of the theory where the typical momentum scale is given by $q_{F}$. For $\delta \ll \gamma$, the dynamic term in the spin fluctuation propagator can be neglected since $\left(q / p_{F}\right)^{2} \sim\left(\Omega / E_{F}\right)^{2} \gg$ $\gamma$. For $\delta \gg \gamma$, the mass of the spin fluctuations can be neglected since $\left(q / p_{F}\right)^{2} \gg \delta$. Thus, in this regime $U \approx\left(p_{F} / q\right)^{2}$. The leading order contributions to $\delta \sigma_{T}(T)$ cancel out, and the triplet channel gives a small contribution to the conductivity $\sigma_{T} \propto 1 / T$. The interference correction is dominated by the contribution from the singlet channel $\delta \sigma \approx \delta \sigma_{S} \propto T$.

In the diffusive limit there are two cross-over regimes (see Fig. 囵).

(1) Regime IV. Setting $\delta=0$, in this regime $U^{-1}\left(\mathbf{q}, \Omega_{n}\right) \approx\left(\left(q / p_{F}\right)^{2}+\left(\gamma\left|\Omega_{n}\right|\right) /\left(D q^{2}\right)\right)$. The leading temperature dependence of the conductivity comes from the triplet channel

$$
\delta \sigma_{T}(T)=\left(3 / 8 \pi^{2}\right)\left(e^{2} \alpha / \gamma\right) \ln ^{2}\left(\gamma D p_{F}^{2} / T\right),
$$

which is guaranteed to win over the singlet one $\left[\delta \sigma_{S}=\right.$ $\left.-\left(e^{2} / 2 \pi^{2}\right) \ln \left(E_{F} / T\right)\right]$ at low enough $T$. This regime has been discussed in the context of $2 \mathrm{D}$ metamagnetic QCP [17], and also in the context of fermion gauge field models 13, 14]. However, our result leads to a metallic sign of the conductivity, which was not noticed in prior work [17]. At finite $\delta$ this regime exists for $T>T_{\delta 2}=\left(\delta^{2} D p_{F}^{2}\right) / \gamma$.

(2) Regime V. For $T<T_{\delta 2}$, the mass of the spin fluctuations is important, and $U^{-1}\left(\mathbf{q}, \Omega_{n}\right) \approx(\delta+$ $\left.\left(\gamma\left|\Omega_{n}\right|\right) /\left(D q^{2}\right)\right)$. The leading temperature dependence of the conductivity in the spin channel is

$$
\delta \sigma_{T}(T)=\left(3 \mathcal{B} / 2 \pi^{2}\right)\left(e^{2} \alpha / \gamma\right) \ln \left(E_{F} / T\right),
$$

where $\mathcal{B}=\ln (\gamma / \delta)$ for $\delta \ll \gamma$, and $\mathcal{B}=\gamma /(2 \delta)$ for $\delta \gg \gamma$. This is the Altshuler-Aronov [1] correction to the conductivity for the triplet channel in the diffusive regime of good metals.

We now turn to the application of our theory to experimental results. In $\mathrm{Sr}_{3} \mathrm{Ru}_{2} \mathrm{O}_{7}$ the velocity $v_{F} / \gamma$ of the spin fluctuations is presumed to be of the order of Fermi velocity, i.e., $\gamma \sim 1$ 17]. Since the in-plane $(\mathrm{ab})$ residual resistivity is $\rho \sim 2.5 \mu \Omega \cdot \mathrm{cm}[10$, and the distance between $\mathrm{RuO}_{2}$ bilayers is $10 \AA$ 24], the residual resistivity per square is $\rho_{2 d} \sim 25 \Omega$. Taking $E_{F} \sim 500 \mathrm{~K}$, we get $1 / \tau \sim 4 \mathrm{~K}$. By comparing the elastic transport rate due to interaction correction $1 / \tau_{\mathrm{el}} \propto \operatorname{Im} \Sigma /\left(v_{F} q \tau\right) \propto T^{1 / 3}$, with the inelastic transport rate due to interaction with the spin fluctuations $1 / \tau_{\text {in }} \propto\left(q / p_{F}\right)^{2} \operatorname{Im} \Sigma \propto T^{4 / 3}$, we expect the quantum correction to be important well below a temperature scale $1 /(\gamma \tau) \sim 4 \mathrm{~K}$. Experimentally, the resistivity is observed to follow $T^{r}$ dependence down to $4 \mathrm{~K}$ with $r \approx 1.2$ [9]. Within our theory, we understand the exponent $r$ as a competition between lattice-mediated inelastic processes above $1 /(\gamma \tau)$ leading to $T^{4 / 3}$ behavior, and quantum interference effects dominating below
$1 /(\gamma \tau)$. We argue that the experimentally observed exponent may be less than $4 / 3$ due to a pre-cursor contribution of the $T^{1 / 3}$ law. Below $4 \mathrm{~K}$ the temperature dependence of conductivity is expected to have the form $\delta \sigma(T)=-a T^{1 / 3}+b T$, where the latter is the regular contribution of the singlet channel 3 . We expect the correction to the conductivity to go from metallic to insulating behavior below $4 \mathrm{~K}$. This could explain the dip in the resistivity observed around $1 \mathrm{~K}$ in this compound $[9]$. Notice that the scale $1 /(\gamma \tau)$ can be increased by increasing disorder.

Conclusions. - Using the spin-fermion model, we studied the quantum interference correction to the conductivity of a 2D disordered itinerant electron system close to a ferromagnetic QCP. Quantum critical fluctuations affect dramatically the temperature dependence of the conductivity, which behaves as $\delta \sigma \propto-T^{1 / 3}$ and $\delta \sigma \propto \ln ^{2} T$ in the ballistic and diffusive regimes respectively. Near the QCP the cross-over temperature between ballistic and diffusive dynamics is $T^{*}=1 /\left(\tau \gamma\left(E_{F} \tau\right)^{2}\right)$. We estimate that quantum intereference dominates the $T$-dependence of $\sigma$ for $T \lesssim 1 / \tau$.

It is our pleasure to thank A. V. Chubukov, Y. B. Kim, A. J. Millis and A. P. Mackenzie for stimulating discussions. The work of D. L. M. was supported by NSF Grant No. DMR-0308377. He also acknowledges the hospitality of the Abdus Salam ICTP.

[1] B. L. Altshuler, and A. G. Aronov, Electron-Electron Interactions in Disordered Systems, edited by A. L. Efros and M. Pollak (North-Holland, Amsterdam, 1985), and references therein.

[2] I. L. Aleiner, B. L. Altshuler, and M. E. Gershenson, Waves Random Media 9, 201 (1999).

[3] G. Zala, B. N. Narozhny, and I. L. Aleiner, Phys. Rev. B 64, 214204 (2001).

[4] G. R. Stewart, Rev. Mod. Phys. 73, 797 (2001).

[5] See e.g., S. R. Julian et al. , J. Phys. Cond. Matter 8, 9675 (1996).

[6] S. Saxena et al., Nature 406,587 (2000); D. Aoki et al., Nature 413,613 (2001); C. Pfleiderer et al., Nature 412, 58 (2001).

[7] T. F. Smith et al., Phys. Rev. Lett. 27, 1732 (1971); M. Uhlarz et al., cond-mat/0408424

[8] D. Sokolov, M. C. Aronson, and Z. Fisk (unpublished).

[9] S. R. Perry et al., Phys. Rev. Lett. 86, 2661 (2001).

[10] S. A. Grigera et al., Science 294, 329 (2001).

[11] A. J. Schofield, A. J. Millis, S. A. Grigera, and G. G. Lonzarich, in Springer Lecture Notes in Physics, 603, 271 (2002).

[12] P. A. Lee, and T. V. Ramakrishnan, Rev. Mod. Phys. 57, 287 (1985).

[13] A. D. Mirlin, and P. Wölfle, Phys. Rev. B 55, 5141 (1997).

[14] D. V. Khveshchenko, Phys. Rev. Lett. 77, 362 (1996); D. V. Khveshchenko, and M. Reizer, cond-mat/9609174

[15] D. Belitz, T. R. Kirkpatrick, M. T. Mercaldo, and S. L. 
Sessions, Phys. Rev. B 63, 174428 (2001).

[16] A. Rosch, Phys. Rev. Lett. 82, 4280 (1999).

[17] Y. B. Kim, and A. J. Millis, Phys. Rev. B 67, 085102 (2003).

[18] J. A. Hertz, Phys. Rev. B 14, 1165 (1976); T. Moriya, Spin Fluctuations in Itinerant Electron Magnetism, (Springer-Verlag, Berlin, New York, 1985); A. J. Millis, Phys. Rev. B 48, 7183 (1993).

[19] D. Belitz, T. R. Kirkpatrick, and T. Vojta, Phys. Rev. B 55, 9452 (1997).
[20] Ar. Abanov, and A. V. Chubukov, cond-mat/0409601

[21] Ar. Abanov, A. V. Chubukov, and J. Schmalian, Adv. Phys. 52, 119 (2003).

[22] A. A. Abrikosov, L. P. Gorkov, and I. E. Dzyaloshinski, Methods of Quantum Field Theory in Statistical Physics, (Dover Publications Inc., New York, 1963).

[23] A. V. Chubukov, C. Pépin, and J. Rech, Phys. Rev. Lett. 92, 147003 (2004).

[24] L. Capogna et al., Phys. Rev. B 67, 012504 (2003). 